\title{
Preventing Distribution Grid Congestion by Integrating Indirect Control in a Hierarchical Electric Vehicles Management System
}

Hu, Junjie; Si, Chengyong; Lind, Morten; Yu, Rongshan

Published in:

IEEE Transactions on Transportation Electrification

Link to article, DOI:

10.1109/TTE.2016.2554469

Publication date:

2016

Document Version

Peer reviewed version

Link back to DTU Orbit

Citation (APA):

Hu, J., Si, C., Lind, M., \& Yu, R. (2016). Preventing Distribution Grid Congestion by Integrating Indirect Control in a Hierarchical Electric Vehicles Management System. IEEE Transactions on Transportation Electrification, 2(3), 290-299. https://doi.org/10.1109/TTE.2016.2554469

\section{General rights}

Copyright and moral rights for the publications made accessible in the public portal are retained by the authors and/or other copyright owners and it is a condition of accessing publications that users recognise and abide by the legal requirements associated with these rights.

- Users may download and print one copy of any publication from the public portal for the purpose of private study or research.

- You may not further distribute the material or use it for any profit-making activity or commercial gain

- You may freely distribute the URL identifying the publication in the public portal 


\title{
Preventing Distribution Grid Congestion by Integrating Indirect Control in a Hierarchical Electric Vehicles Management System
}

\author{
Junjie Hu, Member, IEEE, Chengyong Si, Member, IEEE, Morten Lind and Rongshan Yu, Senior \\ Member, IEEE .
}

\begin{abstract}
In this paper, a hierarchical management system is proposed to integrate electric vehicles (EVs) into a distribution grid. Three types of actors are included in the system: Distribution system operators (DSOs), Fleet operators (FOs) and EV owners. In contrast to a typical hierarchical control system where the upper level controller directly controls the lower level subordinated nodes, this study aims to integrate two common indirect control methods:market-based control and price-based control into the hierarchical electric vehicles management system. Specifically, on the lower level of the hierarchy, the FOs coordinate the charging behaviors of their EV users using a price-based control method. A parametric utility model is used on the lower level to characterize price elasticity of electric vehicles and thus used by the FO to coordinate the individual EV charging. On the upper level of the hierarchy, the distribution system operator uses the market-based control strategy to coordinate the limited power capacity of power transformer with fleet operators. To facilitate the application of the two indirect control methods into the system, a model describing decision tasks in control is used to specify the essential functions that are needed in the control system. The simulations illustrate the effectiveness of the proposed solutions.
\end{abstract}

Index Terms-Congestion prevention, Electric vehicles, Hierarchical control, Indirect control, Price elasticity model

\section{INTRODUCTION}

In addition to their societal and environmental benefits, electric vehicles have been well recognized as a valuable asset to the smart grid in power industry. However, a large scale penetration of electric vehicles may introduce adverse impacts on the secure and economic operation of the power distribution systems if their charging behaviors are not coordinated [1]. Research has been performed to study the EV impacts on the grid from different aspects such as power losses [2], grid congestion and voltage drop [3].

The conclusions are that without charging coordination, the power consumption on a local scale can lead to grid problems as mentioned above. To mitigate the influences, various types of intelligent control strategies have been proposed in the

J. Hu is with the Center for Electric Power and Energy, Department of Electrical Engineering, Technical University of Denmark, Denmark. Email:junhu@elektro.dtu.dk. Junjie is the corresponding author of this paper.

C. $\mathrm{Si}$ is with the Shanghai-Hamburg College, University of Shanghai for Science and Technology (e-mail: sichengyong@gmail.com).

M. Lind is with the Center for Electric Power and Energy, Department of Electrical Engineering, Technical University of Denmark, Denmark. Email: mli@elektro.dtu.dk

R. Yu is with the Signal Processing Department, Institute for Infocomm Research, A*STAR, Singapore. (e-mail: ryu@i2r.a-star.edu.sg). literature. In [4], a smart charging system is designed and implemented that allows the direct interaction between the EVs and power grid. The purpose of the smart charging system is to minimize the charging cost of EVs and prevent damage caused by the excessive loads. In [5], the authors presented a controller at household level based on fuzzy logic to meet the EV charging objective as well as to avoid new peaks consumption. In [6], different priority criteria such as battery state of charge, slack time, and their fairness index are integrated and compared to integrate EV smoothly and efficiently into the distribution network. It is noted that those studies [4]-[6] focus on EV intelligent control strategies design either on the distribution system level or at the household level. However, the FO who will play important role in the near future, is not considered in the studies.

To cover the missing perspective, hierarchical electric vehicles management systems have been proposed and investigated in [7]-[10]. Guille and Gross [7] proposed a framework that recognizes the central role of a EV FO in collecting the EVs to form aggregations and dealing with energy service providers and the system operator for the purchase/provision of energy and capacity services. In [8], a fleet operator is proposed to manage electric vehicles by preparing the buy/sell bids into the electricity market. However, a prior interaction between the FO and the DSO must exist to prevent the occurrence of congestion and voltage problems in the distribution network. In [9], the major objective of the upper-level hierarchy is to minimize the total cost of system operation by jointly dispatching generators and electric vehicle aggregators. The lower-level system aims at strictly following the dispatching instruction from the upper-level decision-maker by designing appropriate charging/discharging strategies for each individual EV in a specified dispatching period. Similar to [9], Xu et al. [10] proposed a hierarchical framework for coordinated charging of electric vehicles in China where the decision made in the upper level is used as a reference in the lower level. In [11], an electric vehicle charging system is envisioned by Ericsson where the electric vehicles charging profiles are coordinated by the aggregator and the utility. The aggregator sends the charging schedule to the EV via public mobile network and the charging logic in the car executes the charging schedule.

It is noted that a limitation can be summarized for above studies. For the proposed hierarchical electric vehicle management system [7]-[11], at each level of the hierarchy, the 
upper level controller directly sends commands in term of power limits, energy dispatching, charging schedule etc to the lower level units. Although the direct control method is widely proposed in both levels of the hierarchical electric vehicles management system, research in [12] indicates that it might not be optimal considering the communication infrastructure cost.

Alternatively, to overcome the limitations of direct control systems, indirect control is proposed and used for EV management [13]-[18]. In [13], [19], two types of indirect control are categorized: market-based control [14], [15] and price-based control [16]-[18]. Market-based control [20] is a paradigm for controlling complex systems with conflicting resources. It typically includes the features found in a market such as decentralized decision making and interacting agents. Marketbased control usually requires two way communication, e.g., exchange the price and power schedule information. Pricebased control, instead, applies broadcasting of price signal with a regular updated frequency to the demand side resources.

Applications of the market-based control in EV charging management are investigated in [14], [15]. The authors assumed that the EVs determine the charging schedule individually with the objective of cost minimization as well as respecting to the energy constraints of driving requirements. Meanwhile, the EVs also need to interact with the upper level controller, i.e., a system operator to achieve the goal of valley filling in such a way that the utility could meet anticipated additional loads for EV charging without additions to their existing resources plan.

In contrast to market-based control, one way communication is used by price-based control. In this scenario, the EV controller does not need to propose and submit their charging profile to the upper level controller. Instead, the upper level controller will anticipate EV response to the price signal sent by it. The price signals ranges from a simple time-ofuse electricity rate [16] to varying hourly prices [17], [18]. The study in [16] suggested that the time-of-use rates can be properly designed to reduce the peak demand as EVs penetrate the vehicle market. It is also noted in [16] that the extent to which properly designed rates could assist in maintaining grid reliability will remain open until EV owners price responsiveness are empirically tested through experiment pilots. Similarly, [17], [18] investigated the price elasticity of general electricity consumers which is considered as one of the key issues in price-based control approach.

It should be noted that all control methods have their limitations. More specifically, direct control implies high communication and computation requirements, price-based control brings higher uncertainty to the controllers such as DSO or FO, market-based control introduces unnecessary complexity to objects under control such as individual EVs as indicated in [14], [15]. To manage and implement the electric vehicles smart charging, the information, interactions, and functions required in fleet operator and EV charging controller are discussed in details in [22] for direct and indirect control method. The charging infrastructure, battery management system, and the communication requirements for smart charging is overviewed in [23]. In order to realize a standardized communication interface between the vehicle and the grid e.g., in Europe, the standardization proposals [24], [25] will make it possible for EV users to have easy access to EV charging equipment (EVSE) and related services throughout Europe. EVSE refers to all devices installed for delivering power from the electrical supply point to the EV and this charging equipment will support smart charging functions. The decision of the charging can be made on the EV level or on the FOs level. The IEC61850 and IEC15118 are the most recommended communication standard, and are demonstrated in details, by the sequence diagram of a charging process between the EVSE and the EVs.

Given the background, this paper aims to integrate marketbased and price-based control strategies in a hierarchical EV management system which is essential to consider the involvements of the FO. The purpose is to use the advantages of the hierarchical electric vehicles management system and the two control methods to ensure the control performance while reducing the communication and computational resource cost.

There are three main research contributions in this study: First, we propose a hierarchical electric vehicle management system to integrate electric vehicles into a distribution network considering the involvement of an important commercial player, i.e., fleet operator in the smart grid. In contrast to a typical hierarchical control system where the upper level controller directly controls the lower level subordinated entities, this study integrates two common indirect control methods: market-based control and price-based control into the hierarchical EV management system. Second, a holistic design procedure is followed in the management system design which includes sketch of the hierarchical electric vehicles charging system, analysis of the system functions and interactions, and control methods development. In particular, the decision model proposed by Rasmussen [26] is applied to specify the functions and interactions for DSO and fleet operator in this hierarchical EV management system. Third, we apply the statistical demand price model previously proposed in [18] to the electric vehicle charging problem in the lower level hierarchy. The impacts of parameter setting on the system is further analyzed to gain better insight on the performance of the proposed system under different application scenarios.

The remainder of the paper is organized as follows: In Section II, a control system architecture is presented which integrates the electric vehicles as well as to prevent the grid congestion. Section III describes the mathematical formulation of the two control algorithms. Simulations are presented in Section IV to illustrate the performance of the proposed control algorithms. Finally, discussion and conclusions are made in Section V.

\section{Control System ARCHItecture DESIGN}

In this section, we firstly introduce the basic concept of hierarchical systems. Then, we apply the concept to design a hierarchical system for integrating EVs into the power distribution systems. Finally, the decision models of DSO and FOs used in the proposed hierarchical electric vehicles management system are described. 


\section{A. A Formal Definition of Hierarchy}

As discussed in [21], a hierarchical system has the following properties:

- A hierarchy has a set of nodes.

- The nodes are connected into a tree structure with a directed relation of subordination.

- A node can only be subordinate to one other node.

- The relation of subordination is transitive (i.e., if Node 1 is subordinate to Node 2, and Node 2 is subordinate to Node 3, then Node 1 is subordinate to Node 3).

- A hierarchy can have any number of levels.

The definition of a hierarchy is dependent on what it means that a node is subordinated to another node. Thus many types of hierarchy could be identified depending on how subordination is defined in the system. In [21], a collection of different types of hierarchy are listed that include control hierarchy, class hierarchy, part-whole hierarchy etc.

\section{B. A Hierarchical Electric Vehicles Management System}

Inspired by the definition of a hierarchy, Fig. 1 presents the architecture of hierarchical electric vehicles management systems which aims to optimally integrate the electric vehicles as well as to prevent the grid congestion. In this system, several FOs are specified to manage the EV fleets, and the distribution system operator can interact with the FOs to eliminate the grid congestion. FO, as a new business entity, is largely proposed in the academy and industry to aggregate electric vehicles charging and exploit new business opportunities by providing multiple services of EVs to system operators. Alternative names for an EV FO are used such as EV virtual power plant, EV aggregator, or EV charging service provider. The new entity [7], [8] could be independent or integrated in an existing business function of the energy supplier or distribution system operator.

We want to point out the difference between a hierarchical system and a control hierarchy. As discussed in section II-A, a control hierarchy system is a type of hierarchical system. A control hierarchy system means the upper level node directly sends commands to the lower subordinated nodes. But in this study, in the upper level hierarchy, the FOs negotiates with the DSO on power transformer capacity instead of specified directly by the DSO. In the lower level hierarchy, the FOs uses the price to coordinate the charging patterns of electric vehicles instead of sending a charging command to the EV fleet. The setup means a coordination hierarchy rather than a control hierarchy is studied in this paper.

Note that the choice of using different control strategies at different levels of the system is based on economic and practical considerations, where the price-based control is used in the lower hierarchy to alleviate the effort of individual EV's operation, while the market-based control used in the upper hierarchy helps DSO to prevent grid congestion in a more accurate way due to a confirmed power schedules of FOs after the negotiations. In addition, it also reduces the computational requirement to DSO because the power schedule decision is made by the FOs.

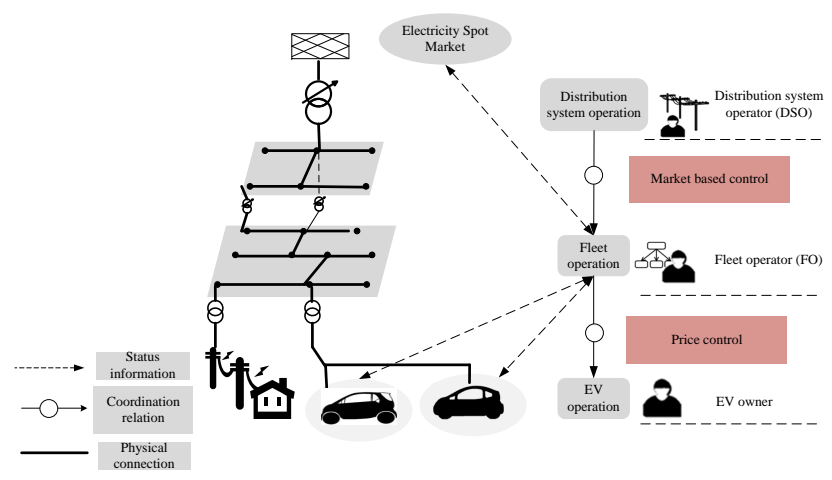

Fig. 1: Power system with electric vehicle integration coordinated by fleet operators

\section{Decision Task Analysis for Distribution System Operator and Fleet Operators}

Fig.1 only gives general relations between different actors in a hierarchical EVs management system and it is unclear how decisions are made for the individual actors in the system. In this section, we present a method to systematically develop the decision models of DSO and FOs. The decision model of EVs is not considered for this study, since their aggregated charging profiles are represented by FOs. Rasmussen's decision model [26] is used to define tasks required in the control system. The model was initially developed for modeling operators decision making in process plant control rooms. As illustrated in Fig. 2 , a separate decision model is used to represent the tasks of DSO and FOs.

1) Decision model for distribution system operator: The actions used in the model include:

- Activate: This function detects the needs for action, for example, the DSO observes the increasing number of EVs that cause overloading of the network in certain period. Then, it will generate altering signals. DSO is activated.

- Observe: When altered, the DSO begins to observe and collect a set of system parameters which form a set of observations.

- Identify: Based on the observations, DSO identifies the system state as normal or abnormal (e.g., part of the network is overloaded in evening time).

- Interpret and Evaluate: According to the system state, DSO will interpret the system considering the performance criteria and thus define the goal state.

Now, it is assumed that the DSO identifies an overloading problem and resolves that it needs to be solved by control actions. The sequence will move to the right branch of the decision model of DSO that defines the control methods.

- Define task: Market-based control is used to resolve the problem.

- Formulate procedure: After identifying the task, the DSO will formulate the procedure to implement the marketbased control, i.e., send price (reflecting congestions) and congestion status (congestion cleared or not) to FOs.

- Execute: When the procedure is ready, DSO will execute the procedure and send the price and congestion status to 


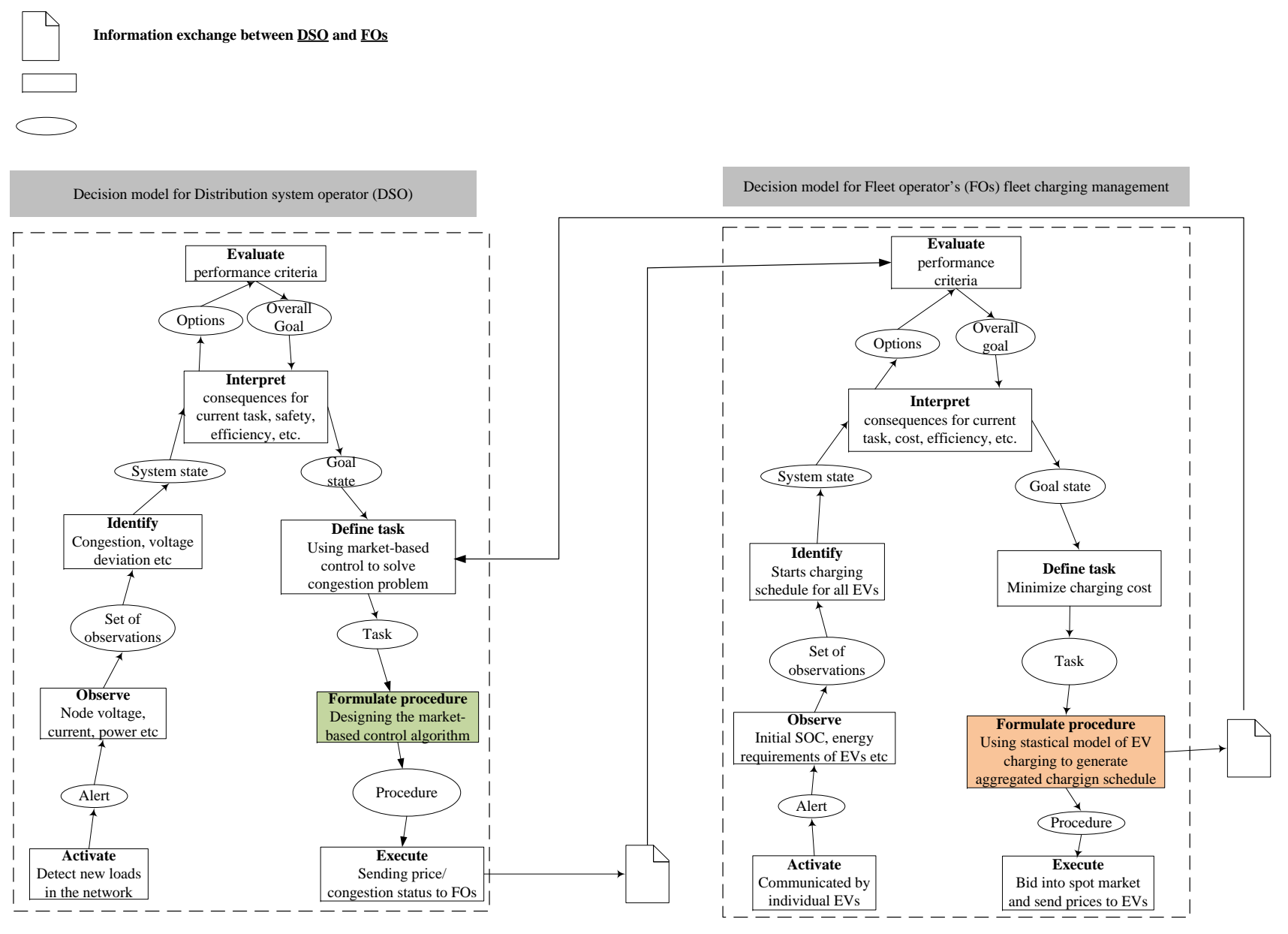

Fig. 2: Decision model for DSO and FO

FOs.

Note that between these two separate decision models of DSO and FOs, information exchanges are needed for coordinations. The prices reflecting grid congestions and the grid congestion status will be sent to FO to influence FOs decision making. FOs send back their aggregated charging schedule to DSOs. The process usually requires a few iterations until the DSO decides to stop the iteration, and signals the FOs to execute the aggregated power schedules.

2) Decision model for fleet operator: The actions in the decision model of FO are described as follows:

- Activate: FO detects the needs for action, e.g., the FO is informed by the EVs.

- Observe: When alerted, the FO begins to observe and collect information and data such as the initial SOC and driving energy requirements of electric vehicles.

- Identify: Based on the observations, FOs identify the system state such as starting the charging schedule preparations.

- Interpret and Evaluate: According to the system state, FO will interpret the system considering the performance criteria and thus define the goal state.

Note that the evaluate block is influenced by the price and congestion status information sent out by the DSO, thus the goal state might be different if nothing is sent by the DSO. In this stage, the goal state is set to minimize the charging cost as well as fulfill the EV users' energy requirements.

- Define task: FO decides to generate optimal charging schedules with the purpose of minimizing the charging cost and therefore avoiding the higher congestion price periods specified by the DSO.

- Formulate procedure: After the task is defined, FOs will formulate the procedure to generate the overall charging schedules using the statistical model of EV charging elasticity. In this block, FOs also need to check the status information and then decide whether they need to send the power schedule to the DSO. This is because in general the congestion periods will be avoided. However, a new congestion might exist if the charging is moving to another period.

- Execute: When the procedure is ready, i.e., the final charging schedule is made. FO will execute the procedure such as bid into spot market and send final price (spot price plus congestion price) to the EVs.

The purpose of this subsection has been to systematically define essential functions and interactions required in the hierarchical electric vehicles charging system when using market-based and price-based control strategies. In section III, 
the focus will be on mathematical modeling of two blocks (the 'Formulate procedure' blocks) defined in the decision models.

\section{Control Methods development}

In this section, we firstly introduce the method used by the FOs to manage the EV fleet charging. The method is used in the 'Formulate procedure' block inside the decision model of FO, highlighted by orange in Fig.2. Then, we present the method for the DSO to design the congestion prices that is used in the 'Formulate procedure' block inside the decision model of DSO, marked as green in Fig.2. In the end, we combine them into an integrated control algorithm that forms a close-loop of this system.

\section{A. Price-based Control Used by FO to Manage the Charging of EV fleet}

In this study, we use the statistical model of demand elasticity developed in [18] and adapt it for EV charging. In general, loads can be classified into three main categories according to their behaviors relative to price: critical loads, interruptible loads and deferrable loads. According to [18], a load is deferrable if the load has non-zero utility values at multiple time slots, which enable the user to reschedule the loads to maximize its benefits considering the timevarying electricity prices. In addition, the load must consume a minimum amount of power over a given interval of time. Normally, the utility function of a deferrable load is at its peak when the load is initially requested, and diminishes over time due to the inconvenience of increasing waiting time to the user. An electric vehicle is a typical load which can be categorized as a deferrable load. Thus the model developed in [18] can be applied to manage a large-scale of electric vehicles and the model is feasible for the FOs to estimate the aggregated EV charging profiles. Note that the model is chosen mainly due to its fitness for the concept of price-based control as well as its effectiveness of obtained an aggregated power profiles. The model predicts EV owners charging preferences over prices without knowing their detailed driving information, then from statistical perspective, the FO can get an aggregated power profiles.

Mathematically, a rational EV owner will choose to schedule his/her charging task to maximize its welfare defined as the difference between its utility and cost:

$$
W_{i}(t)=U_{i}(t)-\rho_{t} * \varphi_{i}(t), t=1, \ldots, T
$$

Dividing (1) by $\varphi_{i}(t)$, one gets

$$
\begin{aligned}
b_{i}(t) & =\frac{U_{i}(t)}{\varphi_{i}(t)}-\frac{\rho_{t} * \varphi_{i}(t)}{\varphi_{i}(t)}, \\
& =r_{i}(t)-\rho_{t}, t=1, \ldots, T
\end{aligned}
$$

where $i=1, \ldots, N_{E}$ is the index of $\mathrm{EV}$ under one FO, $\varphi_{i}(t)$ is the energy/power consumption (hourly based) of $E V_{i}$. $U_{i}(t)$ is the utility function of charging at time $t, \rho_{t}$ is the unit electricity price at time slot $t, r_{i}(t)$ is the marginal utility function of EVs, $b_{i}(t)$ is the unit welfare of the $E V_{i}$ owner.

Accordingly, the EV will be scheduled to the time slots where the welfare function is maximized. One can deduce

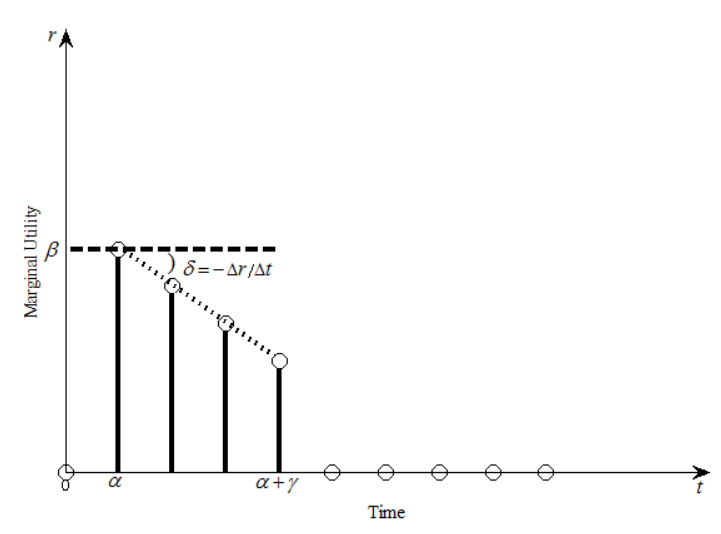

Fig. 3: A marginal utility function of an electric vehicle

that the energy consumption $p_{i}$ of each EV is directly related to the price vector $\boldsymbol{\rho}=\rho_{1}, \ldots, \rho_{T}$ :

$$
p_{i}(\boldsymbol{\rho}, \mathbf{t})= \begin{cases}\varphi_{i}(t), & b_{i}(t)>0, b_{i}(t) \geq b_{i}(\tau), \forall \tau \in \Gamma ; \\ 0, & \text { otherwise }\end{cases}
$$

$\tau$ is the variable for time slot, $\Gamma$ is the set of all time slots, $|\Gamma| \equiv T$. Eq. (3) means if the welfare of $E V i$ reaches a maximum at time slot $t$ (i.e., larger than any other time slot $\tau$ ), the energy consumption of $E V i$ will be $\varphi_{i}(t)$, otherwise, it will be zero.

The sum of energy consumption for all the EVs within one fleet operator, which represents the aggregated demand curve of the fleet operator, is thus a function of electricity price $\rho$, which is given by

$$
P_{t}(\boldsymbol{\rho})=\sum_{i=1}^{N_{E}} p_{i}(\boldsymbol{\rho}, \mathbf{t})
$$

where $P_{t}$ stands for the sum of energy consumptions of all the EVs and $t$ goes from 1 to $T$.

The EV demand of a power network is accordingly completely characterized once the utility functions of all the EVs are known. As illustrated in Fig. 3, it is assumed in [18] that the marginal utility functions of EVs $r_{i}(t)$ is realized by the following parametric stochastic process:

$$
r_{i}(t)= \begin{cases}\beta-\delta(t-\alpha), & \alpha \leq t \leq \alpha+\gamma \\ 0, & \text { otherwise }\end{cases}
$$

where $\alpha, \beta, \gamma, \delta$ are random variables that describe the different characteristics of the utility function:

1) $\alpha$ stands for the time slot that a charging task is initially requested, which also reflects the task distribution;

2) $\beta$ is the initial marginal utility, which stands for the magnitude of the marginal utility;

3) $\gamma$ is the tolerable delay, which determines the maximum delay that a user can tolerate to finish a charging task;

4) $\delta$ is the utility decay rate, which represents the cost of inconvenience by the delay.

The distribution of marginal utility function parameters $\alpha, \beta, \gamma, \delta$ collectively reflects the dynamic behavior of $\mathrm{EV}$ charging. As discussed in [18], these parameters can be 
estimated by survey. Although these parameter values vary from person to person that may be influenced by a number of factors such as users' preferences, economic factors, and other random effects, the aggregated loads can be highly regular for system with a large amount of independent users since it can be guaranteed by the law of large number.

Based on this model, the aggregated demand curve can now be estimated by the expectation with respect to the distribution of $r_{i}(t)$, which is given as follows:

$$
\begin{aligned}
\hat{P}_{t}(\boldsymbol{\rho}) & =\sum_{i=1}^{N_{E}} E\left[p_{i}(\boldsymbol{\rho}, \mathbf{t})\right] \\
& =\sum_{i=1}^{N_{E}} \varphi_{i} * Q\left\{b_{i}(t)>0, b_{i}(t) \geq b_{i}(\tau) ; \forall \tau \in \Gamma\right\}, \\
& =E_{0} * Q(t)
\end{aligned}
$$

where $E[\cdot]$ is stochastic expectation operation, $E_{0}$ is the total EV energy consumption of one FO to be scheduled in the scheduling period, and

$$
Q(t)=Q\left\{b_{i}(t)>0, b_{i}(t) \geq b_{i}(\tau) ; \forall \tau \in \Gamma\right\}
$$

is the probability that a charging will be scheduled at time slot $t$, which can be calculated from the probability distribution $f(\alpha, \beta, \gamma, \delta)$ as follows:

$$
Q(t)=\sum_{\gamma=1}^{\gamma_{\max }} \sum_{\alpha=t-\gamma+1}^{t} \int_{\delta_{t}^{l}}^{\delta_{t}^{h}} \int_{\beta_{t}^{l}}^{\text {inf }} f(\alpha, \beta, \gamma, \delta) d \beta d \delta
$$

Here the upper and lower bounds of the integrals are given by

$$
\begin{aligned}
\beta_{t}^{l} & =\rho_{t}+\delta(t-\alpha) \\
\delta_{t}^{h} & =\min _{u: u \in[t+1, \alpha+\gamma]}\left[\frac{\rho_{t}-\rho_{u}}{u-t}\right] \\
\delta_{t}^{l} & =\max _{u: u \in[\alpha, t-1]}\left[\frac{\rho_{t}-\rho_{u}}{u-t}\right]
\end{aligned}
$$

\section{B. Market-Based Control Used by the DSO to Coordinate the FOs' Charging Schedules}

Denote the aggregated charging schedule $P_{t}$ in (4) as $P_{k, t}$ where $k=1, \ldots, N_{F}$ is the index for the number of FO. For market-based control strategy, different control algorithms can be applied such as a uniform price auction mechanism [27], a shadow-price-based mechanism or a price penalized mechanism [28]. A simple way adapted from [29] is presented in the following:

$$
\Lambda^{\sigma+1}=\Lambda^{\sigma}+\omega * \max \left(0, \frac{P_{b a s e}+\sum_{k=1}^{N_{F}} P_{k, t}-P_{\text {Tran }}^{M a x}}{P_{\text {Tran }}^{M a x}}\right)
$$

where $\Lambda$ is the congestion price that reflects the overloading problem, $\sigma$ is the index for the number of the iterations required between the DSO and the FOs. $\omega$ is the weighting factor. $P_{\text {Tran }}^{M a x}$ denotes the capacity of the power transformer. $P_{\text {base }}$ represents the conventional loads. It can be estimated from the historical loading profiles. The physical meaning of (10) is to determine the congestion price based on the ratio of a power difference (total demand minus power transformer capacity) divided by the power transformer capacity.

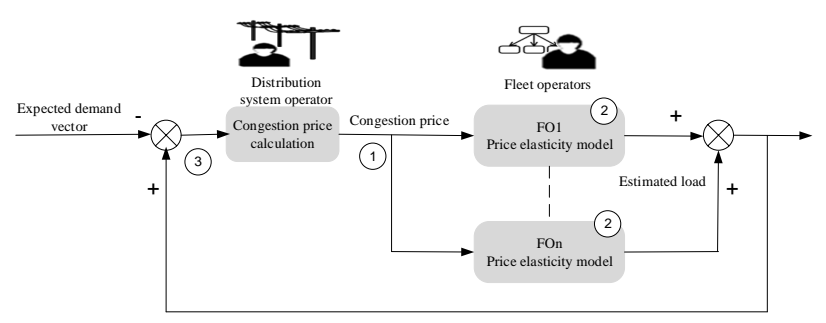

Fig. 4: Scheme of the integrated control algorithm

\section{Integrated Control Algorithm (ICA) for the Hierarchical Electric Vehicles Management System}

As illustrated in Fig. 4, integrated control algorithms used in the hierarchical electric vehicles management system is summarized as following:

- Step1: DSO initiates a congestion price, such as $\Lambda^{0}=0$.

- Step2: FOs uses the congestion price and the predicted spot price to schedule the charging process of the EV fleet. If the congestion status sent out by DSO is flagged as 1, FO resends the aggregated schedule to DSO; otherwise, FOs bid the power schedule into the spot market.

- Step3: DSO checks the grid congestion, updates the congestion status and congestion prices. DSO has the right to determine when to stop the iterative processes, e.g., based on the checked grid status.

- Repeat step 1,2, and 3, until the congestion problem is solved.

The control algorithm described above is stable due to the presence of the feedback loop, which has been shown previously in [14], [15], [30] for systems with similar features. In [14], it is proved that the magnitude of the penalty value for deviating from the mass average has to stay within certain range to reach stable equilibrium. In [15], [30], both studies indicate that even some updated information is missing in some iteration, the stable equilibrium can still be reached. Hence the stability mathematic proof study is omitted here for brevity. Similarly, in this study, as can be seen in (10), $\omega$ plays the role as a penalty parameter when updates the congestion prices. In fact, it is still a challenge to set the penalty parameter effectively in different systems, though many related methods (e.g., static, dynamic, adaptive or selfadaptive penalty function methods) have been proposed [31]. In this study, we focus on demonstrating its effectiveness with simulation results in the next section and analyzing the stability by evaluating some parameter's influences on the control performance.

\section{Case studies}

A typical Danish $10 \mathrm{kV}$ radial network is considered in this case study that supplies 1400 households [32]. We assume that $20 \%$ of the households have an electric vehicle. Two FOs are assumed to manage these $280 \mathrm{EVs}$. FO1 is assumed to manage $180 \mathrm{EVs}$ and FO2 is assumed to manage $100 \mathrm{EVs}$. The time slots considered in the case study range from 16:00 in the afternoon to 6:00 in the morning of next day. It is assumed that the fully charged battery in the morning could sustain 
EVs' daily driving requirements in most time, meaning that in few cases, EVs may need to charge it in other time slots. We assume that FO1 and FO2 share similar information. Among $280 \mathrm{EVs}$, each $20 \%$ are supposed to be charged from $4 \mathrm{kWh}$, $6 \mathrm{kWh}, 8 \mathrm{kWh}, 10 \mathrm{kWh}$ and $12 \mathrm{kWh}$ to $24 \mathrm{kWh}$ respectively. So the total of initial load requested from users will be 2.88 $\mathrm{MWh}$ for FO1 denoted as $E_{1}$ and $1.6 \mathrm{MWh}$ for FO2 denoted as $E_{2}$. And the initial requested load in each time slot will be obtained by $L_{t}=E_{i} * f_{\alpha(t)}, i=1,2$. The probability function of $\alpha$ is set as $f_{\alpha}(t)=\{5 / 39,10 / 39,6 / 39,2 / 39$, $3 / 39,2 / 39,1 / 39,1 / 39,1 / 39,1 / 39,1 / 39,2 / 39,2 / 39,2 / 39\}$. Note the set of $f_{\alpha}$ used here serves an illustrating example, it may changes in different FO EV operation system. In general, the EV users prefer to charge the vehicle as earlier as possible if the electricity price is lower than the utility, so $f_{\alpha}(t)$ has big values in the beginning time slots. For other parameters:

- $\beta, \gamma, \delta$ are assumed to be uniformly distributed within $[1,20],[1,14]$ and $[0.02,1]$, respectively.

- Spot price from the Nordpool market is used and the price information is listed as $\rho_{0}(t)=\{0.3876,0.4734,0.3951$, $0.5338,0.4943,0.4101,0.3774,0.3642,0.3563,0.3514$, $0.3502,0.3498,0.3514,0.3627\}$ DKK/kWh.

- The weighting factor $\omega$ in (10) is 4 .

- The total transformer capacity allocated to two FOs is 1 MW.

A set of experiments are conducted in this section to verify the effectiveness of the proposed integrated algorithms (shown in Section A) and the effect of parameter settings on the performance (presented in Section B). It is important to note that only the results of the time slots $\left(T_{1}, T_{2}\right.$, and $\left.T_{3}\right)$ which can cause difference on the performance of different parameter settings are summarized in this section.

\section{A. Effectiveness of the Proposed Integrated Control Algorithm}

With the above assumptions, the initial requested values of the load in $T_{1}, T_{2}$, and $T_{3}$ are $0.5743 \mathrm{MW}, 1.1487 \mathrm{MW}$, and $0.6892 \mathrm{MW}$, respectively. It is noted that the requested load in $T_{2}$ excesses $1 \mathrm{MW}$. Using (10), we increase the congestion price at $T_{2}$, accordingly, the sum of FOs aggregated demand will decrease. However, as illustrated in Fig. 5, the overloading problem still exists at iteration 1 . Therefore, DSO uses (10) again to increase the congestion price and update the congestion price to two FOs. As shown in Fig. 5, the congestion in $T_{2}$ is solved at iteration 2 .

To better illustrate the effectiveness of the proposed integrated algorithm, we compared ICA with a case using purely price control (PPC), i.e., without the inclusion of marketbased control. In PPC, when solving the peak in $T_{2}$, we set the congestion price 1.5 and 2 times of the original price at iteration 2, respectively. As shown in Fig. 6 and Fig. 7, the congestion in $T_{2}$ can be solved by PPC method (from 1.0853 MW at iteration 1 to $0.5700 \mathrm{MW}$ at iteration 2), however, a new congestion happens (i.e., 1.2680) if the congestion price is set to 2 times of the original price. We can conclude that the effect of purely price control is fast, but the overall performance is hard to control, i.e., how to find the suitable congestion price that does not introduce new peak problem

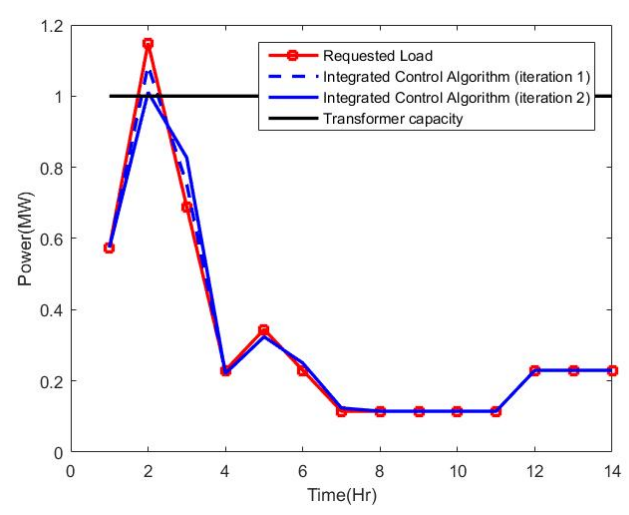

Fig. 5: The impact of the load demand with ICA

[33], [34]. The results indicate an unstable problem if purely price control is applied. From the results, it can be seen that as the proposed integrated control algorithm ICA adopted both the advantage of price control (e.g., quick response) and market based control (e.g., the utilization of feedback information), it could avoid the sharp change on load and show a better overall performance, which verifies the effectiveness of the algorithm.

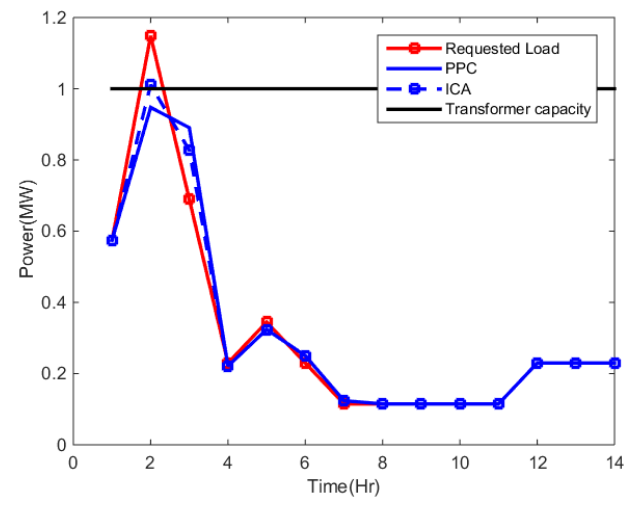

Fig. 6: Comparison of ICA and PPC at iteration 2 when the congestion price of PPC is set as 1.5 times of original spot price

\section{B. Effect of the Parameter Settings}

1) Effect of the weighting factor $\omega$ : Table I summaries the experimental results that only the weighting factor is changed to $1,5,8.9625,13.5$, and 20. From Table I, we can see that if $\omega<5$, the load will decrease and increase gradually in $T_{2}$ and $T_{3}$, as desired; when $\omega \geq 5$, the load will not change during the iterations; especially, when $\omega=8.9625$, the load in $T_{2}$ and $T_{3}$ will be the same. On the other hand, when the weighting factor is relatively larger (i.e., $\omega \geq 13.5$ ), the load goes up and down in both time slots, i.e., oscillation happens.

In addition, Fig. 8, 9, 10 presents the trends for varying $\omega$ at iteration 1 to 3 . In the figures, it can be seen that when $\omega=20$, from iteration 1 to 2 , the load has a sharp decrease 


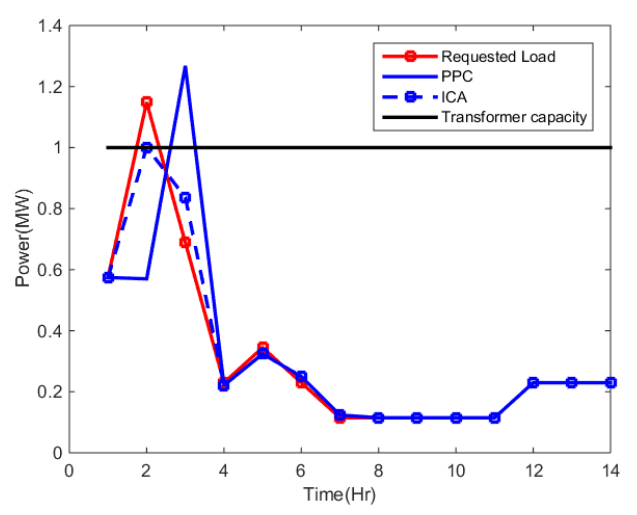

Fig. 7: Comparison of ICA and PPC at iteration 2 when the congestion price of PPC is set as 2 times of original spot price

TABLE I: RESULTS WITH VARYING $\omega$

\begin{tabular}{|c|c|c|c|c|c|}
\hline$\omega$ & 1 & 5 & 8.9625 & 13.5 & 20 \\
\hline Iteration & \multicolumn{5}{|c|}{$T_{2} / \mathrm{MW}$} \\
\hline 1 & 1.0853 & 1.0853 & 1.0853 & 1.0853 & 1.0853 \\
\hline 2 & 1.0667 & 0.9925 & 0.9189 & 0.8347 & 0.7141 \\
\hline 3 & 1.0522 & 0.9925 & 0.9189 & 0.8443 & 1.0278 \\
\hline Iteration & \multicolumn{5}{|c|}{$T_{3} / \mathrm{MW}$} \\
\hline 1 & 0.7527 & 0.7527 & 0.7527 & 0.7527 & 0.7527 \\
\hline 2 & 0.7712 & 0.8455 & 0.9189 & 1.0033 & 1.1239 \\
\hline 3 & 0.7858 & 0.8455 & 0.9189 & 0.9937 & 0.4692 \\
\hline
\end{tabular}

in $T_{2}$ (2 on the x-axis/Time (Hr)) and a sharp increase in $T_{3}$; but from iteration 2 to 3 , there is a sharp increase in $T_{2}$ (even larger than the original load) and a sharp decrease in $T_{3}$. The above results verify that $\omega$ should be chosen with a relatively smaller value.

As can be seen from the results, the weighting factor $\omega$ plays a key role in designing an appropriate congestion price, which will influence the load directly. A too small value will have little effect on the load while a too large value will make the load oscillate. It thus can be concluded that a suitable value of $\omega$ is necessary to balance the system.

Furthermore, the value of $\omega$ will also influence the congestion price, note how to use the price in the reality, e.g., integrated into the electricity bill is out of the scope of this paper but will be addressed in the future work from the authors.

2) Effect of $\beta$ : The experimental results are provided in Table II, with the initial marginal utility $\beta$ being solely changed to [0.1 10], [0.1 20], [0.6 20], [1 20], and [1 10]. As shown in Table II, if the lower boundary of $\beta$ is smaller than a certain value (i.e., 0.6), the load will increase in $T_{2}$ and $T_{3}$ when the upper boundary is increasing; while when the lower boundary of $\beta$ is larger than this value, the load will be kept the same as that in 0.6. This can be concluded that the lower boundary will determine the load directly, while the upper boundary will have some effect on the performance only when the lower boundary is larger than a certain value.

As illustrated in section III. A, $\beta$ stands for the magnitude of the marginal utility, which reflects the welfare or benefits

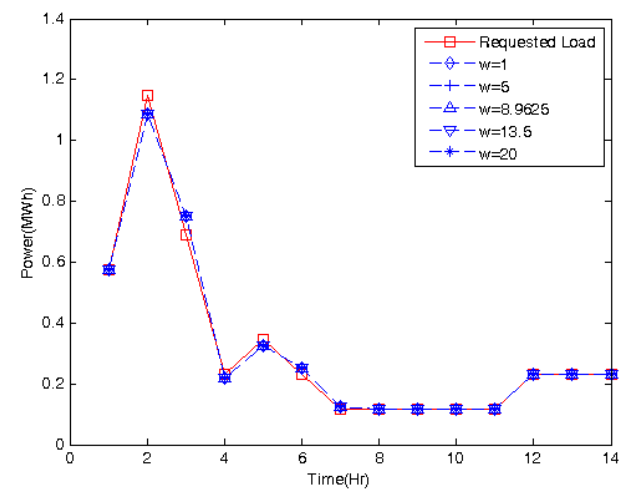

Fig. 8: Comparison of load results with varying $\omega$ at iteration 1

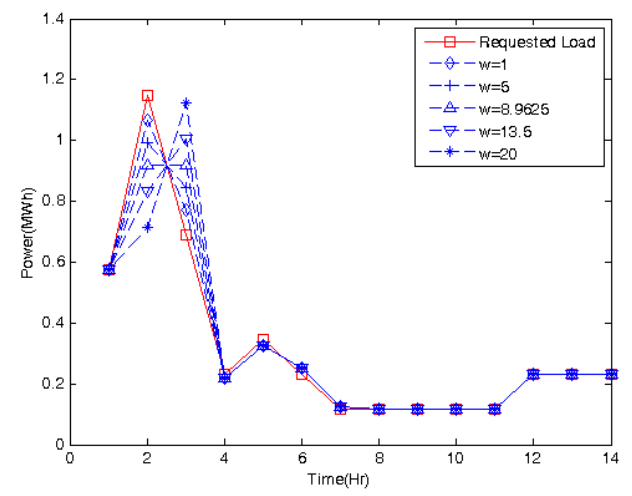

Fig. 9: Comparison of load results with varying $\omega$ at iteration 2

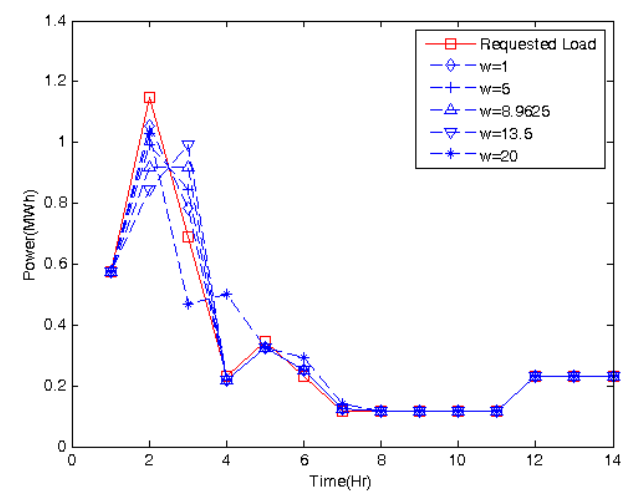

Fig. 10: Comparison of load results with varying $\omega$ at iteration 3 
to a certain degree. So, if the lower boundary is larger than a certain value, i.e., the electricity price, then the price has no influence on the charging schedule, as a smaller value of $\beta$ will cause the EV users to reconsider and reschedule the load in order to maximize the welfare.

TABLE II: RESULTS WITH VARYING $\beta$

\begin{tabular}{|c|c|c|c|c|c|}
\hline$\beta$ & \multirow{2}{*}[\begin{array}{ll}{0.10}\end{array}]{} & {$[0.120]$} & {$[0.620]$} & {$\left[\begin{array}{ll}1 & 20\end{array}\right]$} & {$\left[\begin{array}{ll}1 & 10\end{array}\right]$} \\
\hline Iteration & \multicolumn{5}{|c|}{$T_{2} / \mathrm{MW}$} \\
\hline 1 & 1.0443 & 1.0649 & 1.0853 & 1.0853 & 1.0853 \\
\hline 2 & 1.0034 & 1.0068 & 1.0110 & 1.0110 & 1.0110 \\
\hline 3 & 1.0003 & 1.0007 & 1.0014 & 1.0014 & 1.0014 \\
\hline Iteration & \multicolumn{5}{|c|}{$T_{3} / \mathrm{MW}$} \\
\hline 1 & 0.7299 & 0.7414 & 0.7527 & 0.7527 & 0.7527 \\
\hline 2 & 0.7670 & 0.7967 & 0.8269 & 0.8269 & 0.8269 \\
\hline 3 & 0.7699 & 0.8025 & 0.8365 & 0.8365 & 0.8365 \\
\hline
\end{tabular}

TABLE III: RESULTS WITH VARYING $\delta$

\begin{tabular}{|c|c|c|c|c|c|}
\hline$\delta$ & {$[0.020 .4]$} & {$\left[\begin{array}{ll}0.02 & 1\end{array}\right]$} & {$[0.0210]$} & {$[0.110]$} & {$\left[\begin{array}{ll}1 & 10\end{array}\right]$} \\
& & & & \\
\hline Iteration & \multicolumn{5}{|c|}{$T_{2} / \mathrm{MW}$} \\
\hline 1 & 0.9851 & 1.0853 & 1.1425 & 1.1487 & 1.1487 \\
\hline 2 & 0.9851 & 1.0110 & 1.1303 & 1.1382 & 1.1487 \\
\hline 3 & 0.9851 & 1.0014 & 1.1192 & 1.1263 & 1.1487 \\
\hline Iteration & \multicolumn{5}{|c|}{$T_{3} / \mathrm{MW}$} \\
\hline 1 & 0.8529 & 0.7527 & 0.6955 & 0.6892 & 0.6892 \\
\hline 2 & 0.8529 & 0.8269 & 0.7076 & 0.6997 & 0.6892 \\
\hline 3 & 0.8529 & 0.8365 & 0.7188 & 0.7116 & 0.6892 \\
\hline
\end{tabular}

3) Effect of $\delta$ : Table III presents the experimental results with varying $\delta$, i.e., [0.02 0.4], [0.02 1], [0.02 10], [0.1 10], and $\left[\begin{array}{ll}1 & 10\end{array}\right]$. Table III indicates that if the upper boundary of $\delta$ is smaller than certain value (i.e., 0.4 , in the first column), the load in $T_{2}$ and $T_{3}$ will keep the same; otherwise, the load in $T_{2}$ will decrease and the load in $T_{3}$ will increase as desired when the upper boundary value is increasing (e.g., from 0.4 to 1 and 10). Regarding the lower boundary, if it is larger than a certain value (i.e., 1), the load will also keep the same in both time slots (e.g., the last column). In Table III, it can be seen that the effect of $\delta$ with the range [0.02 1] is more obvious than other ranges. As $\delta$ represents the cost of inconvenience by the delay, the results show that if the cost of inconvenience is too small or too large, the EV users will not be influenced; otherwise, they will reschedule the load according to the cost of inconvenience by the delay.

\section{DISCUSSION AND CONCLUSION}

This paper presents a systematic way of integrating two indirect control methods, i.e., market-based control and pricebased control, into a hierarchical EV management system. It is generally accepted that market-based and price-based control have received a lot of attention in applications to demand response due to their advantages such as lower communication cost and computational complexity. However, both control strategies have the limitations such as price-based control brings higher uncertainty to the controllers while marketbased control introduce complexity to the control objects. The purpose of applying indirect control in a hierarchical electric vehicles management system is to combine the advantages of the two control methods under the framework of hierarchical system. Market-based control strategy is used by the DSO to coordinate with FOs with the purpose of preventing grid congestions by having a confirmed power consumption schedules of FOs at the end of the iterations. In addition, market-based control presents FOs opportunities to negotiate on scarce resources of power transformer. Price control is used by FO to coordinate EV fleets charging behaviors for easy implementation. The statistical model is used in the price control to generate an aggregated power schedule. With the proposed methods, the grid congestion can be solved effectively.

To sum up, the proposed hierarchical EV management system has advantages over purely price-based control (PPC) method, since the PPC control brings oscillations, i.e., new peak loads in the system, while the oscillation problem can be resolved by the proposed system. In addition, compared to the typical hierarchial EV control system, the proposed hierarchical management system keeps privacy to the fleet operators as well as to the EV owners, lowers the computational requirements of power distribution system operators. For future study, it would be interesting to investigate the method of determining the suitable value of omega in real time operation, since it balances the loading profile and the prices. Another interesting research direction is to experimentally test the proposed methods such as via survey, questionnaires etc., with the data collected, the suitable values can be identified in real applications.

\section{REFERENCES}

[1] R. C. Green II, L. Wang, and M. Alam, "The impact of plug-in hybrid electric vehicles on distribution networks: A review and outlook," Renew. Sustain. Energy Rev., pp. 544-553, Jan. 2011.

[2] K. Clement-Nyns, E. Haesen, and J. Driesen, "The impact of charging plug-in hybrid electric vehicles on a residential distribution grid," IEEE Trans. on Power Syst., vol. 25, no. 1, pp. 371-380, 2010.

[3] O. Sundstrom and C. Binding, "Flexible Charging Optimization for Electric Vehicles Considering Distribution Grid Constraints," IEEE Trans. Smart Grid, vol. 3, no. 1, pp. 26-37, 2012.

[4] Abousleiman, R. and Scholer, R., "Smart Charging: System Design and Implementation for Interaction Between Plug-in Electric Vehicles and the Power Grid," IEEE Trans. on Transportation Electrification, vol. 1, pp. 18-25, June 2015.

[5] Berthold, F. and Ravey, A. and Blunier, B. and Bouquain, D. and Williamson, S. and Miraoui, A., "Design and Development of a Smart Control Strategy for Plug-In Hybrid Vehicles Including Vehicle-to-Home Functionality," IEEE Trans. on Transportation Electrification, vol. 2, pp. 168-177, Aug 2015.

[6] Abousleiman, R. and Scholer, R., "Impact of Priority Criteria on Electric Vehicle Charge Scheduling," IEEE Trans. on Transportation Electrification, vol. 3, pp. 200-210, Oct 2015.

[7] C. Guille and G. Gross, "A conceptual framework for the vehicle-to-grid (v2g) implementation," Energy Policy, vol. 37, no. 11, pp. 4379 - 4390, 2009.

[8] J. A. P. Lopes, F. J. Soares, and P. M. R. Almeida, "Integration of electric vehicles in the electric power system," Proc. IEEE, vol. 99, no. 1 , pp. 168-183, 2011. 
[9] W. Yao, J. Zhao, F. Wen, Y. Xue, and G. Ledwich, "A hierarchical decomposition approach for coordinated dispatch of plug-in electric vehicles," IEEE Trans. Power Syst.,, vol. 28, pp. 2768-2778, Aug 2013.

[10] Z. Xu, W. Su, Z. Hu, Y. Song, and H. Zhang, "A hierarchical framework for coordinated charging of plug-in electric vehicles in china," IEEE Trans. on Smart Grid,, vol. PP, no. 99, pp. 1-1, 2015.

[11] I. Olle and F. Anders, "Smart charging for electric vehicles," Energy Theme, 2012.

[12] T. P. Lyon, M. Michelin, A. Jongejan, and T. Leahy, "Is smart charging policy for electric vehicles worthwhile?," Energy Policy, vol. 41, pp. 259-268, 2012.

[13] K. Heussen, S. You, B. Biegel, L. Hansen, and K. Andersen, "Indirect control for demand side management-a conceptual introduction," in $3 r d$ IEEE PES Int. Conf. and Exhib. on Innov. Smart Grid Technol. (ISGT Europe), pp. 1-8, IEEE, 2012.

[14] Z. Ma, D. S. Callaway, and I. A. Hiskens, "Decentralized Charging Control of Large Populations of Plug-in Electric Vehicles," IEEE Trans. Control Syst. Technol., vol. 21, no. 1, pp. 67-78, 2013.

[15] L. Gan, U. Topcu, and S. Low, "Optimal decentralized protocol for electric vehicle charging," IEEE Trans. Power Syst., vol. 28, no. 2 , pp. 940-951, 2013.

[16] S. Shao, T. Zhang, M. Pipattanasomporn, and S. Rahman, "Impact of TOU rates on distribution load shapes in a smart grid with PHEV penetration," IEEE PES Transm. Distrib. Conf. Expo.: Smart Solutions for a Changing World, 2010.

[17] O. Corradi, H. Ochsenfeld, H. Madsen, and P. Pinson, "Controlling Electricity Consumption by Forecasting its Response to Varying Prices,' IEEE Trans. Power Syst., vol. 28, no. 1, pp. 421-429, 2013.

[18] R. Yu, W. Yang, and S. Rahardja, "A Statistical Demand-Price Model With Its Application in Optimal Real-Time Price," IEEE Trans. on Smart Grid, vol. 3, no. 4, pp. 1734-1742, 2012.

[19] J. Hu, S. You, C. Si, M. Lind, and J. Østergaard, "Optimization and control method for smart charging of evs facilitated by fleet operator: review and classification," Int. Journal of Distributed Energy Resources, vol. 10 , pp. 383-397, 2013.

[20] S. H. Clearwater, Market based control: a paradigm for distributed resource allocation. World Scientific, 1996.

[21] M. Lind, "Hierarchies/heterarchies, note for course hierarchical control and automation systems.," tech. rep., Technical University of Denmark, 2014.

[22] J. Garca-Villalobos, I. Zamora, J.I SanMartn, F.J. Asensio, V. Aperribay, "Plug-in electric vehicles in electric distribution networks: a review of smart charging approaches," Renew Sustain Energy Rev, vol. 38, pp. 717-731, 2014.

[23] W. Su, H. Eichi, W. Zeng, M. Chow, "A Survey on the Electrification of Transportation in a Smart Grid Environment," IEEE Trans. Ind. Informat, vol. 8, no. 1, pp. 1-10, 2012.

[24] S. Kabisch , A. Schmitt, M. Winter, J. Heuer, "Interconnections and communications of electric vehicles and smart grids," 1st IEEE Int. Conf. on smart grid communications (SmartGridComm), p. 161166, 2010.

[25] K. Hansch, A. Pelzer, P. Komarnicki, S. Groning, J. Schmutzler, C. Wietfeld, J. Heuer, R. Muller, "An ISO/IEC15118 conformance testing system architecture," Proc. of the IEEE PES General Meeting Conf. Expo, p. 15, 2014.

[26] J. Rasmussen, Information Processing and Human-Machine Interaction: An Approach to Cognitive Engineering. New York, NY, USA: Elsevier Science Inc., 1986.

[27] R. D. Zimmerman, "Uniform price auctions and optimal power flow," Matpower Technical Note, Feb, vol. 1, 2010

[28] J. Hu, S. You, M. Lind, and J. Ostergaard, "Coordinated charging of electric vehicles for congestion prevention in the distribution grid," IEEE Trans. Smart Grid, vol. 5, pp. 703-711, March 2014.

[29] R. A. Verzijlbergh, L. J. De Vries, and Z. Lukszo, "Renewable energy sources and responsive demand. do we need congestion management in the distribution grid?," IEEE Trans. Power Syst., vol. 29, no. 5, pp. 2119$2128,2014$.

[30] N. Gatsis and G. Giannakis, "Residential load control: Distributed scheduling and convergence with lost ami messages," IEEE Trans. Smart Grid, vol. 3, pp. 770-786, June 2012.

[31] A. Eiben, R. Hinterding, and Z. Michalewicz, "Parameter control in evolutionary algorithms," IEEE Trans. Evol. Comput., vol. 3, no. 2, pp. 124141, July 1999.

[32] J. Østergaard, and J. Nielsen, "The bornholm power system an overview," 2008.

[33] A. Pentland, "Economics: Simple market models fail the test," Nature 525,190191 (10 September 2015).
[34] SM. Krause, S. Börries, S. Bornholdt, "Econophysics of adaptive power markets: When a market does not dampen fluctuations but amplifies them," Phys. Rev. E92, 012815 (2015).

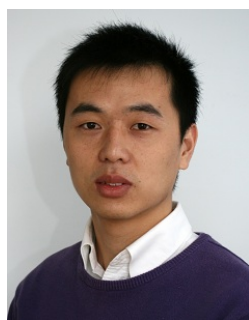

Junjie Hu (M'2014) received the Ph.D degree in Electrical Engineering from Technical University of Denmark. Currently, he is a Postdoctoral researcher within the the Department of Electrical Engineering within the Technical University of Denmark (DTU). His main fields of interests are distributed energy resources integration with focus on electric vehicles, application of optimal control theory on active distribution grid operation and management.

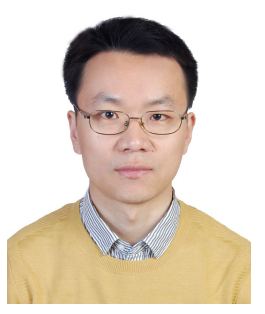

Chengyong Si (M'2014) received the Masters degree in control theory and control engineering from Shanghai Maritime University (SMU), Shanghai, the $\mathrm{Ph} . \mathrm{D}$. degree in Control theory and Control Engineering from Tongji University, Shanghai, China, in 2009 and 2013, respectively. He is currently a lecturer with Shanghai-Hamburg College, University of Shanghai for Science and Technology (USST), Shanghai, China. He was a research scholar with Institute for Electronics Engineering, University of Erlangen-Nuernberg. His current research includes evolutionary computation, constrained optimization, multiobjective optimization, and their applications.

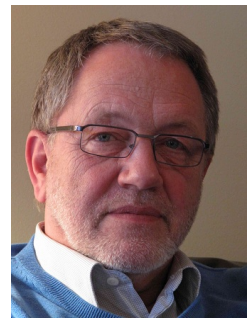

Morten Lind is Professor Emeritus of Automation at Department of Electrical Engineering at Technical University of Denmark and is associated with Centre for Electric Power and Energy. His research interests include automation design, supervisory control of complex industrial systems and infrastructures, functional modeling and application of agent technology and knowledge based system in automation.

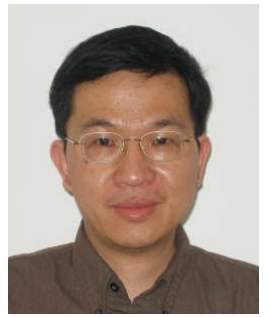

Rongshan Yu (M'01,SM'05) received his B.Eng degrees in electrical engineering with minor in applied mathematics from Shanghai Jiaotong University in 1995, and PhD degree in electrical engineering from the National University of Singapore, Singapore in 2004. In 1999, he joined the Centre for Signal Processing, Nanyang Technological University, Singapore as a Research Engineer. After that, he joined Dolby Laboratories, USA as a staff engineer in 2006. Dr. Yu joined Institute for Infocomm Research (I2R), Agency for Science, Technology and Research (A*STAR), Singapore as a research scientist in 2009 and currently he is the Head of Signal Processing Department. His research interests include statistical signal processing, smart grid and computer architectures for big data processing. He is a Senior Member of IEEE, and Fellow of IET. 